Gut, 1984, 25, 1085-1088

\title{
Significance of basement membrane thickening in the human colon
}

\author{
ANN GLEDHILL AND F M COLE \\ From the Department of Anatomic Pathology, McMaster University Medical Centre, Hamilton, Ontario, \\ Canada
}

SUMMARY Collagenous colitis is a rare condition characterised by watery diarrhoea. It is thought to be caused by a thick collagenous membrane found immediately beneath the colonic surface epithelium. We have studied specimens from 457 patients with a wide variety of large bowel diseases in order to determine the frequency, nature, and clinical correlations of the thick membranes. The normal membrane measured up to 3 microns. In $19(4 \%)$ of the patients studied there was significant thickening (greater than $10 \mu$ ). In 12 patients the thickening ranged from $10-15 \mu$, and six of this group had diarrhoea. In seven, the membrane exceeded $15 \mu$, and six patients had diarrhoea. There was no correlation between the nature of the disease process, bleeding, pain, constipation or previous medication. It did not vary with the age, nor at different sites of the colon. We concluded that no case in this series represented an example of true collagenous colitis but that significant thickening of the membrane occurs in other disease conditions and that a thick membrane, no matter how it arises, is causally associated with profuse watery diarrhoea.

In 1965, Lindström ${ }^{1}$ described a patient who presented with watery diarrhoea of unknown cause. Colonic biopsy was unremarkable apart from a prominent linear eosinophilic thickening immediately below the surface epithelium. It was suggested that this membrane caused the diarrhoea by acting as a barrier to water absorption from the large bowel. Lindström regarded this as a distinct entity for which he coined the term 'collagenous colitis'. Since then, there have been about 12 other cases $^{2-9}$ recorded in the English literature, together with a recent comprehensive review, ${ }^{10}$ all showing similar clinical and pathological features.

Judging from the paucity of reports, the condition would appear to be rare and poorly understood. For this reason, we have attempted to characterise in more detail the features of the membrane thickening, its nature, whether the degree of thickening varies in different areas of the large bowel or with advancing age, and whether it is associated with any particular disease. Further studies were made to determined the normal range in a control group.

Address for correspondence: Dr F M Cole, Chief of Service, Anatomic Pathology, McMaster University Medical Centre, 1200 Main St. W, Hamilton, Ontario, Canada L8N3Z5

Received for publication 11 January 1984

\section{Methods}

\section{BIOPSIES}

A retrospective study over a period of $2 \frac{1}{2}$ years was carried out on consecutive colonic biopsies (365) and resection specimens (92) from 457 patients. In most of the patients undergoing biopsies, multiple specimens were obtained from various areas of the large bowel and these sites were documented. The patients covered an age range from 2 months to 94 years, and had presented to the gastroenterology and surgery departments with a wide range of complaints referable to the gastrointestinal tract.

Tissue was fixed in $10 \%$ formol saline, paraffin embedded, and $5 \mu$ section stained with haematoxylin and eosin (H \& E), McManus periodic acid-Schiff reagent (PAS), van Gieson's collagen fibre stain (VG), Jones' methenamine silver (JMS), Masson's Trichrome (TRI), and Congo red. Portions of selected specimens were fixed in glutaraldehyde, post fixed in osmium tetroxide and stained with uranyl acetate, embedded in epoxy resin and sections examined under a transmission electron microscope.

Using the $\mathrm{H} \&$ E slides, an initial semiquanititative grading of the membrane thickness on a $0-4$ 
scale was carried out by both pathologists independently. Accurate measurements of those graded 2, 3 and 4 were obtained by 20 point counts using a calibrated eye piece graticule. The 20 point counts were performed on 10 adjacent intercryptal areas. Two readings on each intercryptal subepithelial membrane were measured.

Necropsy material was obtained from 47 patients dying unexpectedly, usually because of road traffic accidents. Thirty seven were unsuitable owing to autolysis of the superficial mucosa, the value for the normal range being derived from the remaining 10 .

\section{Results}

\section{FREQUENCY AND EXTENT OF MEMBRANE}

THICKENING

The thickness of the membrane in the normal individuals varied from $0-3 \mu$. In the symptomatic group, there was wide variation. In the majority (267 of 457) no membrane was discernible. In 171 it was thickened but to less than $10 \mu$, in 12 to between 10 and $15 \mu$ and in the remaining seven it exceeded $15 \mu$, the maximum being $22.5 \mu$. These are all mean values; focal areas of up to $80 \mu$ were seen in some specimens. These results are summarised in the Table.

\section{MORPHOLOGICAL FEATURES OF THE MEMBRANE THICKENING}

The membrane is situated immediately below the intercryptal epithelium, extending only a short distance into the crypt (Figure). The membranes in the control group were of uniform thickness throughout the large bowel. Many of the patients with membrane thickening had multiple simultaneous biopsies and these together with resected specimens showed similar values at all sites.

Congo red stains were consistently negative. Staining with PAS and JMS was variable, apart from a thin line of strongly positive material immediately below the epithelium, corresponding to the lamina densa. The whole band stained positive for collagen with Masson's trichrome and van Gieson's stain. Birefringence was present in about half of the cases; it appeared to depend upon the density and spatial arrangement of the collagen fibres.

Table Distribution of membrane thickening

\begin{tabular}{llllll}
\hline $\begin{array}{l}\text { Total } \\
\text { no. of } \\
\text { patients }\end{array}$ & $\begin{array}{l}\text { Patients with } \\
\text { no membrane } \\
\text { thickening }\end{array}$ & $\begin{array}{l}\text { Patients with } \\
\text { thickening } \\
<10 \mu\end{array}$ & $10-15 \mu$ & $>15 \mu$ \\
\hline 457 & 267 & 171 & 12 & 7 \\
\hline
\end{tabular}

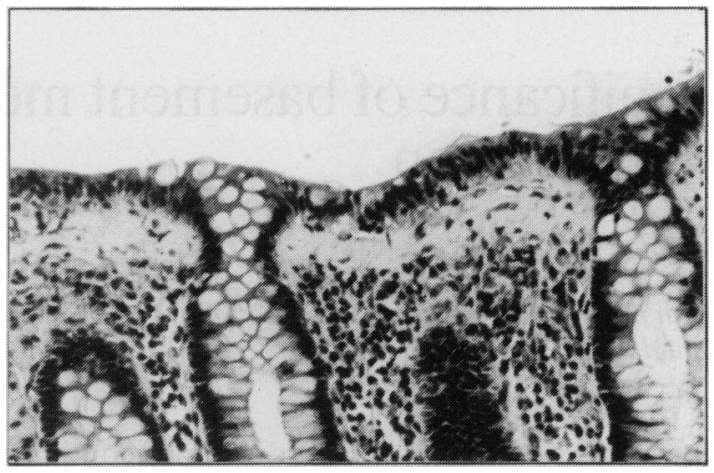

Figure Collagenous band situated immediately beneath the colonic surface epithelium. The mean thickness of the membrane in this case was $16 \mu$ ( $H \&$ E 250 original magnification).

Electron microscopy showed that the membrane thickening was caused by increased amounts of collagen and basement membrane like amorphous material. There were no amyloid or immune complex deposits.

\section{CLINICAL AND PATHOLOGICAL CORRELATION}

The disease processes of those patients with membrane thickening over $10 \mu$ included nonspecific colitis (four), adenocarcinoma (six), megacolon (two), epithelial polyps (six) and diverticular disease (one). There was no correlation with age, the range being 18-90 years, nor with other features such as medication, rectal bleeding or pain. Of the seven patients with membrane thickening over $15 \mu$, however, all but one presented with profuse watery diarrhoea. Of 12 with membranes measuring 10-15 $\mu$, six had diarrhoea.

As an incidental finding, some degree of thickening was invariably seen within hyperplastic polyps, whereas it was not seen within adenomatous polyps or adenocarcinoma. It was occasionally a feature of the otherwise normal bowel adjacent to, but remote from, adenocarcinoma. Surprisingly, it was not observed in any case of proven chronic inflammatory bowel disease.

\section{Discussion}

There have been about 12 cases of collagenous colitis reported in the English literature since 1976, all presenting with diarrhoea and the histological finding of a subepithelial collagen band varying in thickness from 11.5-60 $\mu$. In the original description, Lindström ${ }^{1}$ gave no measurements, but the illustrations suggest a thickness of approximately 
$15 \mu$. The lowest value, $11.5 \mu$, was recorded by Bogomoletz, ${ }^{2}$ but is probably the most accurate, as this was a mean value derived from 20 point counts. Other workers state either the maximum thickness or performed only single measurements. Early in our study, it became apparent that if only occasional measurements were taken, serious overestimations could result, as despite focal areas with thicknesses of up to $80 \mu$ the overall value dropped to around 20 $\mu$ when the mean of multi-point measurements was calculated.

Difficulties have been encountered in the past in assessing the normal range. Bogomoletz attempted to establish a figure by taking as a control 30 biopsies reported as 'being within normal limits'. Precisely what is meant by normal limits is unclear. Furthermore, it seems probable that all these came from patients with symptoms referable to the bowel, and as such, could not be regarded as entirely normal. Kaye et $a^{11}$ derived their normal range from the resection margins of colons removed for adenocarcinoma. This probably results in too great a value, as in our studies thickening was often seen adjacent to carcinoma. We felt that the best control group would consist of otherwise healthy people who died suddenly, as in road traffic accidents, and therefore feel that our value of up to $3 \mu$ is correct.

In assessing the incidence of significant thickening, we chose the arbitrary figure of greater than $10 \mu$ as this represented at least a three-fold increase over the norm. Even in patients with a wide variety of bowel disorders, many longstanding, this proved to be a rare finding. This is in agreement with the findings of other observers ${ }^{7}$ who examined 564 specimens from patients with a variety of conditions, and found six with membrane thickening. These authors felt that the thickening in these cases was of no significance as it was patchy. Our findings indicate that when present, it was diffuse, independent of the disease process, age, and medication. The only associated symptom was diarrhoea, six out of seven patients with thickening over $15 \mu$ presenting with diarrhoea. These findings indicate that the presence of the collagen band in itself, for whatever cause, is likely to be associated with watery diarrhoea. All previous authors have suggested that the collagen band reduces water and electrolyte resorption from the large bowel, thus resulting in diarrhoea. Its occurrence in otherwise unrelated conditions, with no other associated symptoms, supports this view. Despite the diversity of disease states, it is possible that there is a common mechanism underlying its formation.

Pascal et al ${ }^{12}$ have shown that around the crypts of the normal colon lies a sheath of pericryptal fibroblasts. These are closely applied to the epithelial lamina densa, their replication and migration being linked to that of the overlying epithelial cells with active division in the crypt base, migration to the luminal surface being accompanied by maturation. Fibroblasts tend not to produce collagen during active reproduction, hence in the normal colon little collagen is seen around the lower half of the crypt, the bulk being produced by the mature fibrocytes lying under the intercryptal epithelium.

In a situation where cell turnover is increased for whatever reason, the reproductive phase of the fibrocyte would predominate over the mature fibrogenic phase, and collagen production would decrease. This would explain why the collagen band was frequently not visible in active inflammation, within carcinoma, or in adenomatous polyps. Conversely, if cell turnover decreased, each fibrocyte would spend longer in the mature phase, hence more collagen would be produced, resulting in a thicker collagen table. The factor causing the reduced turnover could vary in differing disease states, but produce similar symptoms with a similar histological picture.

We thank Dr Gerard Simon for his help with the electron microscopy and Miss Mary McNeil for typing the manuscript.

\section{References}

1 Lindström CG. Collagenous colitis with watery diarrhea - a new entity? Pathol Eur 1976; 11: 87-9.

2 Bogomoletz WV, Adnet JJ, Birembaut P, Feydy P, Dupont $P$. Collagenous colitis: an unrecognised entity. Gut 1980; 21: 164-8.

3 Neilson VT, Vetner M, Harsløf E. Collagenous colitis. Histopathology 1980; 4: 83-6.

4 Pieterse AS, Hecker R, Rowland R. Collagenous colitis: a distinctive and potentially reversible disorder. $J$ Clin Pathol 1982; 35: 338-40.

5 Stubbe Teglbjaerg P, Hess Thaysen E. Collagenous colitis: an ultrastructural study of a case. Gastroenterology 1982; 82: 561-3.

6 Hansen J, Henrik-Nielsen R, Grove O, Raskmadsen J. Collagenous colitis: an unrecognised cause of secretory diarrhea. Scand J Gastroenterol 1981; 16: 1112.

7 Van den Oord JJ, Geboes K, Desmet VJ. Collagenous colitis: an abnormal collagen table? Two new cases and a review of the literature. Am J Gastroenterol 1982; 77: 377-81.

8 Grouls V, Vogel J, Sorger M. Collagenous colitis. Endoscopy 1982; 14: 31-3.

9 Bamford MJ, Matz LR, Armstrong JA, Harris ARC. Collagenous colitis: a case report and review of the literature. Pathology 1982; 14: 481-4. 
10 Bogomoletz WV. Collagenitis colitis: a clinicopathological review. Surv Dig Dis 1983; 1: 19-25.

11 Kaye GI, Lane N, Pascal RR. Colonic pericryptal fibroblast sheath; replication, migration and cytodifferentiation of a mesenchymal cell system in adult tissue. II; fine structural aspects of normal rabbit and human colon. Gastroenterology 1968; 54: 852-65.

12 Pascal RR, Kaye GI, Lane N. Colonic pericryptal fibroblast sheath; replication, migration and cytodifferentiation of a mesenchymal cell system in adult tissue. I; autoradiographic studies of normal rabbit colon. Gastroenterology 1968; 54: 835-51. 